\title{
La depreciación del peso mexicano durante 20I2-2015 y su efecto en el Índice de Precios y Cotizaciones de la Bolsa Mexicana de Valores. Un análisis intersectorial
}

\author{
Depreciation of the Mexican peso during 2012-2015 and its effect on
} the Index of Prices and Quotations (IPC) of the Mexican Stock Exchange.

\section{Resumen}

El peso mexicano tuvo una depreciación de más del 30\% en el último lustro, históricamente las disminuciones de la moneda nacional han tenido un impacto negativo en las empresas nacionales con transacciones en divisa extranjera.Esta investigación muestra los efectos de la depreciación del peso mexicano frente al dólar en las empresas que integran el Índice de Precios y Cotizaciones (IPC) de la Bolsa Mexicana de Valores (BMV), con un enfoque intersectorial, de acuerdo a la clasificación de sectores de la BMV durante 2012-2015. Para evaluar los efectos en las corporaciones se usaron tres múltiplos financieros que miden: (1) la depreciación cambiaria, (2) las ganancias cambiarias, y (3) el nivel de pasivos en moneda extranjera, y para calcular el impacto en cada uno de los sectores se usó un análisis estadístico de varianza (ANOVA), con un nivel de confianza del 95\%, y con ello examinar si existe diferencia estadística en los valores promedio en los múltiplos financieros prevalecientes entre los sectores. Los resultados señalan que el efecto fue diferente para cada sector en lo que se refiere a los rubros de pérdidas cambiarias y nivel de deuda en moneda extranjera, sin embargo en el renglón de las ganancias cambiarias el efecto fue similar para todos los sectores.

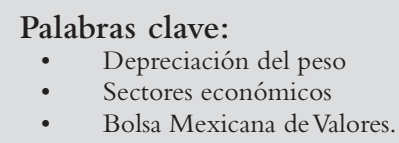

\author{
José Antonio Morales Castro* \\ María Fernanda Velázquez Chávez** \\ Cinthia Elvia García Rodríguez***
}

* Docente investigador en la Escuela Superior de Comercio y Administración unidad Tepepan del Instituto Politécnico Nacional y miembro del Sistema Nacional de Investigadores del Consejo Nacional de Ciencia y

Tecnología, correo electrónico: jmorales@ipn.mx. **Integrante del programa formación de investigadores del Instituto Politécnico Nacional. 


\section{Introducción}

El objetivo de este trabajo es mostrar los efectos de la depreciación del peso mexicano frente al dólar en las empresas que integran índice de precios y cotizaciones (IPC) de la Bolsa Mexicana de Valores (BMV), con un matiz intersectorial, de acuerdo a la clasificación de sectores de la BMv durante periodo de 2012-2015, mediante un análisis de varianza (ANOVA), en los múltiplos de ganancias y pérdidas cambiarias.

En México a finales de diciembre de 1994 el tipo de cambio era muy volátil, en noviembre se encontraba en 3.43 pesos por dólar, cerrando en 4.99, para el mes de enero de 1995 alcanzó valores de 5.90 pesos por dólar, en ese momento su devaluación fue de $89.99 \%$ respecto al año anterior, esta crisis implico el abandono del régimen de bandas cambiarias para México y la adopción del régimen de libre flotación del peso mexicano (Nájera y Gutiérrez, 2013).

"Se ha argumentado que los sistemas de flotación cambiaria pueden ser más
riesgosos que los sistemas de paridades fijas, es decir, se espera que la paridad
de los tipos de cambios cuando hay libre flotación sea mayor pues se permite
que la paridad esté modificándose de manera prácticamente constante" (Ló-
pez, Rodríguez y Ortiz, 2011:20).

El precio del peso mexicano se ha depreciado más del $30 \%$ durante el último lustro, es a partir del año 2014 cuando el precio de la moneda nacional ha descendido drásticamente en términos de dólares, es decir, hay que pagar más por cada dólar, en el año de 2011 se encontraba en 12.00 pesos por cada dólar, llegando hasta niveles de casi 17.0 pesos para el segundo semestre de 2015. El aumento de precio del dólar tiene efectos nocivos para las empresas que compran en el extranjero materia prima, maquinaria, diversos insumos y servicios, porque se traduce en mayores costos de producción, que se trasladan a los consumidores finales aumentado el precio de venta, por una parte, por otra lado disminuyen los resultados financieros de las empresas, al aumentar los gastos financieros para la compra de dólares que se necesitan al solventar las obligaciones en moneda extranjera. 
- $\square$ - José Antonio Morales Castro, María Fernanda Velázquez Chávez y Cinthia Elvia García Rodríguez

Las empresas que cotizan en la Bolsa Mexicana de Valores, realizan operaciones comercio y financieras con el extranjero, por ello resulta que:

Estudiar el tipo de relaciones que envuelven al mercado cambiario y al mercado bursátil, es importante porque históricamente los movimientos de ambos mercados han generado los peores desastres económicos y financieros en nuestro país, al menos en las tres últimas décadas, (Guzmán, Leyva y Cárdenas, 2007: 83).

Por otra parte la globalización caracterizada en términos de la expansión del comercio exterior entre los países explotando las ventajas comparativas de cada uno de ellos, con la finalidad de obtener mayores ingresos, la inversión de los inversores en otro país distinto a su país de origen, empresas con filiales operando en varios países, individuos que trabajan en países distintos al que nacieron (Krugman y Wells, 2013; Mankiw, 2015 y Madura, 2015), estos son aspectos que caracterizan actualmente a las empresas de la BMV, debido a que han aumentado su presencia en otros países en los últimos años mediante el incremento de exportaciones, establecimiento de sucursales, acrecentamiento de líneas de productos, lo cual tiende a fortalecerlas comercial y financieramente, la depreciación del peso mexicano no las ha afectado como en las depreciaciones y devaluaciones pasadas, es lo que se estudia en esta investigación.

\section{Impacto de las variaciones del valor de la divisa nacional en las corporaciones}

Cuando el precio de una divisa varía en relación a las de otros países, tiene efectos diferentes tanto en las empresas como en los diversos rubros de la balanza comercial de los países. En lo que se refiere al análisis corporativo, la economía financiera analiza las ventajas y desventajas del uso de recursos ajenos -deuda-sobre los recursos propios - acciones- a la hora de financiar las inversiones (Marín y Rubio, 2001: 10), de las compañías en sus operaciones, tanto rutinarias como estratégicas.

Tradicionalmente se piensa que una depreciación cambiaria otorga un nivel de competitividad de corto plazo a las exportaciones, esperando que disminuya un déficit comercial o que se incremente el superávit, si es que lo hay. Por lo tanto una apreciación de tipo cambio, incrementa el poder adquisitivo del peso en el exterior, haciendo que se deteriore la balanza comercial (Reyes, Moslares y Sotomayor, 2012: 27). 
En las compañías que tienen pasivos en moneda extranjera, cuando ocurre una depreciación de la moneda nacional se convierte en pérdidas cambiarias, las cuales se producen al momento de pagar los pasivos en moneda extranjera, las compañías erogan más cantidad de moneda nacional por cada unidad de divisa extranjera, estas pérdidas cambiarias deterioran las ganancias obtenidas por las empresas y viceversa, cuando se aprecia la divisa nacional con relación a las monedas extranjeras, se producen ganancias cambiarias, porque se pagan menos cantidad en moneda nacional por las divisas extranjeras que se necesitan para solventar los pasivos en moneda extranjera que las firmas tienen documentados.

Una depreciación de la divisa nacional abarata las exportaciones, por consecuencia aumentan las ventas de las empresas nacionales, por otra parte, en el caso de las empresas que poseen activos en moneda extranjera dentro de su balance general, como es el caso de las cuentas por cobrar en moneda extranjera, una depreciación de la moneda nacional se reflejará en una ganancia cambiaria, pues al convertir las divisas recibidas del extranjero en moneda nacional, éstas tendrán mayor valor en términos de la moneda local, convirtiéndose en ganancia cambiaria para la corporación, y cuando la moneda nacional se aprecia frente a las divisas extranjeras, se traducirá en una perdida cambiaria, al convertir los ingresos provenientes del extranjeros en moneda nacional.

\section{Estudios empíricos}

En lo que se refiere a la relación del comportamiento del tipo de cambio con el sector bursátil en México, están los estudios de Guzmán, Leyva y Cárdenas, (2007), Guzmán y Padilla (2009), Reyes, Moslares y Sotomayor, (2012), Sosa y Cabello (2015).

Guzmán y Padilla (2009), estudió el impacto de la política monetaria sobre la tasa de interés, el tipo de cambio y su relación con los rendimientos del índice bursátil, encontró que los efectos entre ambas variables son pequeños y se desvanecen a lo largo de siete días, mediante el análisis de la descomposición de la varianza muestra que después de diez días, el porcentaje de variación de la tasa de interés, del tipo de cambio y el índice bursátil debida a que la variación del saldo objetivo es menor a 2.0\%, llega a la conclusión que este porcentaje muestra que el cambio en la postura de política monetaria del Banco de México, sobre las variables en estudio, es pequeña y se desvanece rápidamente. 
Guzmán, Leyva y Cárdenas, (2007) señalan que durante 2003-2006, los movimientos del mercado bursátil estuvieron atados al comportamiento de los mercados financieros internacionales, principalmente de Estados Unidos; y por su parte, los movimientos del mercado cambiario respondieron a la mayor oferta de dólares derivada del incremento en el precio del petróleo y del aumento de remesas.

Sosa y Cabello (2015), estudiaron la relación de las variables macroeconómicas con los mercados accionarios del grupo BRICS, Corea del Sur, Indonesia, Turquía y México, determinando el riesgo sistemático para estos mercados, tomando en consideración cambios en cuatro variables macroeconómicas: índice de precios al consumidor, producción industrial, volumen de exportaciones y reservas internacionales; como variables explicativas de los principales índices bursátiles para cada economía, durante el periodo 2003-2013.

Reyes, Moslares y Sotomayor, (2012), explican la afectación de la volatilidad cambiaria a las exportaciones durante 2002-2010 a través de un estudio empírico, encentraron que cada año se comportó distinto, existen años en que la correlación es negativa, es decir, que las exportaciones cayeron aun cuando el tipo de cambio se depreció, los productos que parecen reaccionar de manera más sensible al tipo de cambio nominal en el corto plazo son las exportaciones en los rubros de agricultura y silvicultura y ganadería, apicultura y pesca, donde suponen que la apreciación del peso estimula la producción y venta externa de productos agrícolas y del bosque, productos ganaderos, abejas y pesca. "El grueso del comercio con el exterior está condicionado por el crecimiento económico, mismo que a su vez está estrechamente correlacionado con el de los Estados Unidos” (Reyes, Moslares y Sotomayor, 2012: 48).

\section{Planteamiento de hipótesis}

Las empresas reflejan contablemente las consecuencias de las depreciaciones y apreciaciones del peso mexicano frente a otras divisas extranjeras, en los rubros de ganancias y pérdidas cambiarias, los cuales miden los impactos de las variaciones del precio del peso mexicano en los corporativos (CINIF, 2015), y según estudios empíricos, "No todos los sectores se comportan igual, como en el caso de las manufacturas, que dependen más de la actividad económica de los Estados Unidos que de variaciones en el tipo de cambio nominal" (Reyes, Moslares y Sotomayor, 2012: 27), y a pesar de que existen otros estudios como son los de Guzmán, Leyva y Cárdenas, (2007), Guzmán y Padilla (2009), Sosa y Cabello (2015), no existe un análisis de los efectos de la de- 
preciación del peso mexicano frente al dólar durante 2012-2015 y su secuela en cada uno de los sectores de acuerdo a la clasificación de sectores de la BMV durante periodo de 2012-2015, para ello se plantean las siguientes hipótesis de investigación.

\section{Hipótesis}

$H_{0}$ : Los valores de los ratios financieros que miden las pérdidas cambiarias son iguales para todos los sectores de empresas del IPC: $\mu_{1}=\mu_{2}=\mu_{3}=\mu_{4}=\mu_{5}=\mu_{6}$.

$H_{1}$ : Alguno de los sectores tiene valores diferentes en su múltiplo de pérdida cambiaria.

Se plantean las mismas hipótesis para los ratios que miden la ganancia cambiaria y el nivel de deuda en moneda extranjera.

\section{Metodología de la investigación}

El IPC de la BMV está conformado por 35 empresas, de las cuales se excluyeron las compañías del sector financiero, porque su contabilidad es distinta a las empresas de producción-comercialización tradicionales, después se obtuvieron los estados financieros de ECONOMATICA para las 32 empresas restantes cuadro 1, con los cuales se calcularon tres múltiplos financieros: (1) pérdidas cambiarias a utilidad neta (PCaUN), (2) ganancias cambiarias a utilidad neta (GCaUN) y (3) pasivo en moneda extranjera a pasivo total (PMEaPT), por el periodo que comprende del primer trimestre de 2012 al segundo trimestre de 2015. Posteriormente se hizo un análisis de varianza (ANOvA) para probar si las medias de los tres múltiplos financieros entre las empresas difieren según el sector al que pertenecen, con la finalidad de evaluar el impacto de la depreciación del peso en cada uno los sectores de la BMV.

El ANOva permite contrastar la igualdad de medias de tres o más poblaciones (Pérez, 2004; Hair, Anderson, Tatham y Black, 1999; Haeussler, Paul y Wood, 2008), en este caso se comparan los valores medios de los múltiplos (pérdidas cambiarias, ganancias cambiarias y deuda en moneda extranjera) para los seis sectores de la BMV. ${ }^{1}$ Esta técnica requiere de tomar una muestra aleatoria simple independiente de cada uno de los sectores que conforman el IPC, y se requiere que las poblaciones muestreadas estén normalmente distribuidas y con varianzas idénticas. "Resulta que la prueba de análisis de va-

\footnotetext{
${ }^{1}$ En este caso se ha denominado a los sectores: 1. Telecomunicaciones, 2. Productos de consumo frecuente, 3. Materiales, 4. Industrial, 5. Servicios de consumo no frecuente, 6. Salud.
} 
- $\square=\square$ José Antonio Morales Castro, María Fernanda Velázquez Chávez y Cinthia Elvia García Rodríguez

rianza es bastante robusta con respecto a la suposición de normalidad (incluso desviaciones moderadas de esta suposición no cambian mucho los resultados), pero cualquier violación de la suposición de varianzas iguales afecta seriamente la validez de la prueba" (Kohler, 1998: 484). Donde se calculan los estimadores $S_{T}^{2}$ y $S_{R}^{2}$ y el valor del estadístico $\frac{S_{T}^{2}}{S_{R}^{2}}$, considerando:

$S_{R}^{2}=\frac{\sum_{i}^{k} \sum_{j}^{n_{i}}\left(\bar{y}_{t i}-\bar{y}_{i}\right)^{2}}{N-k} S_{T}^{2}=\frac{\sum_{i}^{k} n_{i}\left(\bar{y}_{t i}-\bar{y}\right)^{2}}{k-1}$

Posteriormente se calcula el valor de $F_{k-1, n-k}$ para el nivel de significación prefijado, si: $\frac{S_{T}^{2}}{S_{R}^{2}}>F_{k-1, n-k}$ se considera que la diferencia es estadísticamente significativa, si $\frac{S_{t}^{2}}{S_{s}^{2}}<F_{k-1, n-k}$, la diferencia no es estadísticamente significativa (Hair, Anderson, Tatham y Black, 1999 y Heussler, 2008).

\section{Cuadro I}

\section{Empresas del Índice de Precios y} Cotizaciones de la Bolsa Mexicana de Valores

\begin{tabular}{|c|c|c|c|}
\hline 1 & AMX & AMERICA MOVIL, S.A.B. DE C.V & $\begin{array}{l}\text { Servicios } \\
\text { de telecomunicaciones }\end{array}$ \\
\hline 2 & WALMEX & WAL - MART DE MEXICO, S.A.B. DE C.V. & $\begin{array}{l}\text { Productos } \\
\text { de consumo frecuente }\end{array}$ \\
\hline 3 & FEMSA & $\begin{array}{l}\text { FOMENTO ECONÓMICO MEXICANO, S.A.B. } \\
\text { DE C.V. }\end{array}$ & $\begin{array}{l}\text { Productos } \\
\text { de consumo frecuente. }\end{array}$ \\
\hline 4 & TLEVISA & GRUPO TELEVISA, S.A.B. & $\begin{array}{l}\text { Servicios } \\
\text { de telecomunicaciones }\end{array}$ \\
\hline 5 & GMEXICO & GRUPO MEXICO, S.A.B. DE C.V. & Materiales. \\
\hline 6 & CEMEX & CEMEX, S.A.B. DE C.V. & Materiales. \\
\hline 7 & ALFA & ALFA, S.A.B. DE C.V. & Industrial \\
\hline 8 & GMODELO & EMPRESA DESLISTADA EL 12 MAYO DEL 2015. & $\begin{array}{l}\text { Productos } \\
\text { de consumo frecuente. }\end{array}$ \\
\hline 9 & PE\&OLES & INDUSTRIAS PEÑOLES, S. A.B. DE C.V. & Materiales \\
\hline 10 & $\mathrm{KOF}$ & COCA-COLA FEMSA, S.A.B. DE C.V. & $\begin{array}{l}\text { Productos } \\
\text { de consumo frecuente. }\end{array}$ \\
\hline 11 & ELEKTRA $\star$ & GRUPO ELEKTRA, S.A.B. DE C.V. & $\begin{array}{l}\text { Servicios y bienes } \\
\text { de consumo no básico }\end{array}$ \\
\hline 12 & MEXCHEM $\star$ & MEXICHEM, S.A.B. DE C.V. & Materiales. \\
\hline 13 & BIMBO A - & GRUPO BIMBO, S.A.B. DE C.V. & $\begin{array}{l}\text { Productos } \\
\text { de consumo frecuente. }\end{array}$ \\
\hline 14 & $\mathrm{AC}^{\star}$ & ARCA CONTINENTAL, S.A.B. DE C.V. & $\begin{array}{l}\text { Productos } \\
\text { de consumo frecuente. }\end{array}$ \\
\hline
\end{tabular}


Economía Informa núm. 397 marzo - abril • 2016

\begin{tabular}{|c|c|c|c|}
\hline 15 & KIMBER & KIMBERLY - CLARK DE MEXICO S.A.B. DE C.V. & $\begin{array}{l}\text { Productos } \\
\text { de consumo frecuente }\end{array}$ \\
\hline 16 & $\mathrm{LAB}$ & $\begin{array}{l}\text { GENOMMA LAB INTERNACIONAL, S.A.B. DE } \\
\text { C.V. }\end{array}$ & Salud \\
\hline 17 & MFRISCO & MINERA FRISCO, S.A.B. DE C.V. & Materiales \\
\hline 18 & LIVEPOL & EL PUERTO DE LIVERPOOL, S.A.B. DE C.V. & $\begin{array}{l}\text { Servicios y bienes } \\
\text { de consumo no básico }\end{array}$ \\
\hline 19 & ASUR & $\begin{array}{l}\text { GRUPO AEROPORTUARIO DEL SURESTE, } \\
\text { S.A.B. DE C.V. }\end{array}$ & Industrial. \\
\hline 20 & GAP & $\begin{array}{l}\text { GRUPO AEROPORTUARIO DEL PACIFICO, } \\
\text { S.A.B. DE C.V. }\end{array}$ & Industrial. \\
\hline 21 & ALPEK & ALPEK, S.A.B. DE C.V. & Materiales \\
\hline 22 & $\mathrm{ICA}^{\star}$ & EMPRESAS ICA, S.A.B. DE C.V. & Industrial. \\
\hline 23 & $\mathrm{ICH}$ & INDÚSTRIAS CH, S.A.B. DE C.V. & Materiales \\
\hline 24 & AZTECA & TV AZTECA, S.A.B. DE C.V. & $\begin{array}{l}\text { Servicios } \\
\text { de telecomunicaciones }\end{array}$ \\
\hline 25 & OHLMEX ${ }^{\star}$ & OHL MEXICO, S.A.B. DE C.V. & Industrial. \\
\hline 26 & GEO B & CORPORACION GEO, S.A.B. DE C.V. & Industrial. \\
\hline 27 & HOMEX` & DESARROLLADORA HOMEX, S.A.B. DE C.V. & Industrial. \\
\hline 29 & GRUMA & GRUMA, S.A.B. DE C.V. & $\begin{array}{l}\text { Productos } \\
\text { de consumo frecuente }\end{array}$ \\
\hline 30 & ALSEA $\star$ & ALSEA, S.A.B. DE C.V. & $\begin{array}{l}\text { Servicios y bienes } \\
\text { de consumo no básico }\end{array}$ \\
\hline 31 & CHDRAUI & GRUPO COMER CIAL CHEDRAUI, S.A.B. DE C.V. & $\begin{array}{l}\text { Productos } \\
\text { de consumo frecuente }\end{array}$ \\
\hline 32 & URBI* - & URBI DESARROLLOS URBANOS, S.A.B. DE C.V. & Industrial. \\
\hline
\end{tabular}

\section{Resultados del estudio empírico}

Se usó el programa SPSS (Pérez, 2004) para procesar los datos y así obtener los estadísticos descriptivos y el ANOvA correspondiente, donde se obtuvieron los siguientes resultados, los cuales se presentan en los cuadros 2, 3, 4, 5, 6, 7, 8 y 9. En los cuadros 2, 6 y 8 se presentan los estadísticos descriptivos de los múltiplos PCaUN, GCaUN y PMEaPT, respectivamente por sector al que pertenecen de las empresas.

En lo que se refiere al múltiplo PCaUN en el cuadro 3, se presenta el estadístico de Levene el cual es menor a 0.05 , por lo cual se rechaza la hipótesis de igualdad de varianzas, en el cuadro 4 se presenta el nivel de significancia del estadístico F, el cual es menor a .05, por lo cual se rechaza la hipótesis de igualdad de medias. 
Cuadro 2

Estadísticos descriptivos del múltiplo pérdidas cambiarias a utilidad neta

\begin{tabular}{|c|c|c|c|c|c|c|c|c|}
\hline \multirow{3}{*}{ PCaUN } & \multicolumn{8}{|c|}{ Descriptivos } \\
\hline & \multirow{2}{*}{$\mathrm{N}$} & \multirow{2}{*}{ Media } & \multirow{2}{*}{$\begin{array}{l}\text { Desviación } \\
\text { típica }\end{array}$} & \multirow{2}{*}{$\begin{array}{l}\text { Error } \\
\text { típico }\end{array}$} & \multicolumn{2}{|c|}{$\begin{array}{c}\text { Intervalo de } \\
\text { confianza para la } \\
\text { media al } 95 \% \\
\end{array}$} & \multirow{2}{*}{ Mínimo } & \multirow{2}{*}{ Máximo } \\
\hline & & & & & $\begin{array}{l}\text { Límite } \\
\text { inferior }\end{array}$ & $\begin{array}{l}\text { Límite } \\
\text { superior }\end{array}$ & & \\
\hline $\begin{array}{l}\text { 1. Telecomunica- } \\
\text { ciones }\end{array}$ & 42 & .2839 & .70082 & .10814 & .0655 & .5023 & -.39 & 2.80 \\
\hline $\begin{array}{l}\text { 2. Produc. consumo } \\
\text { frecuente }\end{array}$ & 126 & .0693 & .24897 & .02218 & .0254 & .1132 & 0.00 & 2.45 \\
\hline 3. Materiales & 98 & .0850 & .41489 & .04191 & .0018 & .1682 & -1.64 & 1.53 \\
\hline 4. Industrial & 112 & -.0689 & .38079 & .03598 & -.1402 & .0024 & -2.13 & .84 \\
\hline $\begin{array}{l}\text { 5. Serv. y bienes } \\
\text { cons. no frecuente }\end{array}$ & 42 & .0146 & .18270 & .02819 & -.0424 & .0715 & -.63 & .84 \\
\hline 6. Salud & 14 & .1123 & .15426 & .04123 & .0233 & .2014 & 0.00 & .53 \\
\hline Total & 434 & .0541 & .39190 & .01881 & .0171 & .0910 & -2.13 & 2.80 \\
\hline
\end{tabular}

Fuente: Resultados de la investigación.

\section{Cuadro 3}

\section{Prueba de homogeneidad de varianzas de pérdidas cambiarias}

\begin{tabular}{|c|c|c|c|}
\hline $\begin{array}{c}\text { Estadístico de } \\
\text { Levene }\end{array}$ & g11 & g12 & Sig. \\
\hline 7.621 & 5 & 428 & .000 \\
\hline
\end{tabular}

Fuente: Resultados de la investigación.

\section{Cuadro 4}

\section{Prueba de la significancia de la ANOva}

\begin{tabular}{|c|c|c|c|c|c|}
\hline \multicolumn{6}{|c|}{ PCaUN. ANOva de un factor } \\
\hline & Suma de cuadrados & g1 & Media cuadrática & $\mathbf{F}$ & Sig. \\
\hline Inter-grupos & 4.148 & 5 & .830 & 5.695 & .000 \\
\hline Intra-grupos & 62.355 & 428 & .146 & & \\
\hline Total & 66.503 & 433 & & & \\
\hline
\end{tabular}

Fuente: Resultados de la investigación. 
Cuadro 5

Comparaciones entre los sectores de empresas de la BMV pérdidas cambiarias

\begin{tabular}{|c|c|c|c|c|c|c|c|}
\hline \multicolumn{3}{|c|}{$\begin{array}{c}\text { Variable dependiente: } \\
\text { PCaUN }\end{array}$} & \multicolumn{5}{|c|}{ Comparaciones múltiples } \\
\hline \multirow{2}{*}{\multicolumn{3}{|c|}{ (I) Sector }} & \multirow[b]{2}{*}{$\begin{array}{l}\text { Diferencia de } \\
\text { medias (I-J) }\end{array}$} & \multirow{2}{*}{$\begin{array}{l}\text { Error } \\
\text { típico }\end{array}$} & \multirow[b]{2}{*}{ Sig. } & \multicolumn{2}{|c|}{ Intervalo de confianza al $95 \%$} \\
\hline & & & & & & $\begin{array}{c}\text { Límite } \\
\text { inferior }\end{array}$ & $\begin{array}{c}\text { Límite } \\
\text { superior }\end{array}$ \\
\hline \multirow[t]{30}{*}{ Scheffé } & 1.00 & 2.00 & .21461 & .06801 & .079 & -.0127 & .4420 \\
\hline & & 3.00 & .19893 & .07039 & .160 & -.0364 & .4343 \\
\hline & & 4.00 & $.35281 \star$ & .06906 & .000 & .1219 & .5837 \\
\hline & & 5.00 & .26937 & .08329 & .065 & -.0091 & .5478 \\
\hline & & 6.00 & .17159 & .11779 & .832 & -.2222 & .5654 \\
\hline & 2.00 & 1.00 & -.21461 & .06801 & .079 & -.4420 & .0127 \\
\hline & & 3.00 & -.01568 & .05141 & 1.000 & -.1875 & .1562 \\
\hline & & 4.00 & .13820 & .04957 & .172 & -.0275 & .3039 \\
\hline & & 5.00 & .05476 & .06801 & .986 & -.1726 & .2821 \\
\hline & & 6.00 & -.04302 & .10753 & .999 & -.4025 & .3164 \\
\hline & 3.00 & 1.00 & -.19893 & .07039 & .160 & -.4343 & .0364 \\
\hline & & 2.00 & .01568 & .05141 & 1.000 & -.1562 & .1875 \\
\hline & & 4.00 & .15388 & .05280 & .134 & -.0226 & .3304 \\
\hline & & 5.00 & .07044 & .07039 & .962 & -.1649 & .3058 \\
\hline & & 6.00 & -.02734 & .10906 & 1.000 & -.3919 & .3372 \\
\hline & 4.00 & 1.00 & $-.35281^{\star}$ & .06906 & .000 & -.5837 & -.1219 \\
\hline & & 2.00 & -.13820 & .04957 & .172 & -.3039 & .0275 \\
\hline & & 3.00 & -.15388 & .05280 & .134 & -.3304 & .0226 \\
\hline & & 5.00 & -.08344 & .06906 & .917 & -.3143 & .1474 \\
\hline & & 6.00 & -.18122 & .10820 & .730 & -.5429 & .1805 \\
\hline & 5.00 & 1.00 & -.26937 & .08329 & .065 & -.5478 & .0091 \\
\hline & & 2.00 & -.05476 & .06801 & .986 & -.2821 & .1726 \\
\hline & & 3.00 & -.07044 & .07039 & .962 & -.3058 & .1649 \\
\hline & & 4.00 & .08344 & .06906 & .917 & -.1474 & .3143 \\
\hline & & 6.00 & -.09778 & .11779 & .983 & -.4916 & .2960 \\
\hline & 6.00 & 1.00 & -.17159 & .11779 & .832 & -.5654 & .2222 \\
\hline & & 2.00 & .04302 & .10753 & .999 & -.3164 & .4025 \\
\hline & & 3.00 & .02734 & .10906 & 1.000 & -.3372 & .3919 \\
\hline & & 4.00 & .18122 & .10820 & .730 & -.1805 & .5429 \\
\hline & & 5.00 & .09778 & .11779 & .983 & -.2960 & .4916 \\
\hline
\end{tabular}

Fuente: Resultados de la investigación.

En el cuadro 5 se presentan la prueba de las diferencias de medias entre los grupos (sectores de la BMV), en este caso se identifica por la columna de sig- 
$\square=\square$ José Antonio Morales Castro, María Fernanda Velázquez Chávez y Cinthia Elvia García Rodríguez

nificación, si su valor es menor o igual a 0.05, expresa que las diferencias formadas entre los sectores que se combinan tienen diferencias significativas en el valor medio del ratio pérdidas cambiarias a utilidad neta PCaUN.

Para el caso de las ganancias cambiarias GCaUN el estadístico de Levene es menor a 0.037 , por lo cual se rechaza la hipótesis de igualdad de varianzas, el nivel de significancia del estadístico F, es 0.880 , el cual es mayor que 0.05, por lo cual se acepta la hipótesis de igualdad de medias.

\section{Cuadro 6}

\section{Estadísticos descriptivos del múltiplo ganancias cambiarias a utilidad neta}

\begin{tabular}{|c|c|c|c|c|c|c|c|c|}
\hline \multicolumn{9}{|c|}{ GCaUN. Descriptivos } \\
\hline & \multirow[t]{2}{*}{$\mathrm{N}$} & \multirow[t]{2}{*}{ Media } & \multirow{2}{*}{$\begin{array}{l}\text { Desviación } \\
\text { típica }\end{array}$} & \multirow{2}{*}{$\begin{array}{l}\text { Error } \\
\text { típico }\end{array}$} & \multicolumn{2}{|c|}{$\begin{array}{c}\text { Intervalo de } \\
\text { confianza para la } \\
\text { media al } 95 \%\end{array}$} & \multirow[t]{2}{*}{ Mínimo } & \multirow[t]{2}{*}{ Máximo } \\
\hline & & & & & $\begin{array}{l}\text { Límite } \\
\text { inferior }\end{array}$ & $\begin{array}{l}\text { Límite } \\
\text { superior }\end{array}$ & & \\
\hline $\begin{array}{l}\text { 1. Telecomunica- } \\
\text { ciones }\end{array}$ & 42 & .1077 & .26032 & .04017 & .0265 & .1888 & 0.00 & 1.37 \\
\hline $\begin{array}{l}\text { 2. Produc. consumo } \\
\text { frecuente }\end{array}$ & 126 & .0931 & .29015 & .02585 & .0420 & .1443 & -.01 & 2.32 \\
\hline 3. Materiales & 98 & .0882 & .65368 & .06603 & -.0429 & .2192 & -5.03 & 2.53 \\
\hline 4. Industrial & 112 & .0893 & .58571 & .05534 & -.0203 & .1990 & -1.75 & 5.13 \\
\hline $\begin{array}{l}\text { 5. Serv. y bienes } \\
\text { cons. no frecuente }\end{array}$ & 42 & .0060 & .11962 & .01846 & -.0312 & .0433 & -.48 & .58 \\
\hline 6. Salud & 14 & .0016 & .00601 & .00161 & -.0019 & .0051 & 0.00 & .02 \\
\hline Total & 434 & .0810 & .46544 & .02234 & .0371 & .1250 & -5.03 & 5.13 \\
\hline
\end{tabular}

Fuente: Resultados de la investigación.

En el cuadro 7 se presentan la prueba de las diferencias de medias de las ganancias cambiarias entre los grupos (sectores de la BMV), donde la columna de significación es mayor a 0.05 , lo cual simboliza que los sectores que se combinan no tienen diferencias estadísticamente significativas, es decir, sus valores medios son casi iguales para los seis sectores, tal como se muestra en el cuadro 6. 


\section{Cuadro 7}

\section{Comparaciones entre los sectores de empresas} de la BMV ganancias cambiarias

\begin{tabular}{|c|c|c|c|c|c|c|c|}
\hline \multicolumn{8}{|c|}{ Variable dependiente: GCaUN. Comparaciones múltiples } \\
\hline & \multirow{2}{*}{\multicolumn{2}{|c|}{ (I) Sector }} & \multirow{3}{*}{$\begin{array}{c}\begin{array}{c}\text { Diferencia } \\
\text { de medias } \\
(\mathrm{I}-\mathrm{J})\end{array} \\
.01454\end{array}$} & \multirow{3}{*}{$\begin{array}{l}\text { Error } \\
\text { típico }\end{array}$} & \multirow{3}{*}{$\begin{array}{l}\text { Sig. } \\
1.000\end{array}$} & \multicolumn{2}{|c|}{\begin{tabular}{|c|} 
Intervalo de \\
confianza al $95 \%$ \\
\end{tabular}} \\
\hline & & & & & & \multirow{2}{*}{\begin{tabular}{|c|}
$\begin{array}{c}\text { Límite } \\
\text { inferior }\end{array}$ \\
-.2637 \\
\end{tabular}} & \multirow{2}{*}{$\begin{array}{c}\text { Límite } \\
\text { superior } \\
.2928\end{array}$} \\
\hline Scheffé & 1.00 & 2.00 & & & & & \\
\hline & & 3.00 & .01950 & .08616 & 1.000 & -.2685 & .3075 \\
\hline & & 4.00 & .01832 & .08453 & 1.000 & -.2643 & .3009 \\
\hline & & 5.00 & .10160 & .10195 & .963 & -.2392 & .4424 \\
\hline & & 6.00 & .10605 & .14418 & .990 & -.3759 & .5880 \\
\hline & 2.00 & 1.00 & -.01454 & .08324 & 1.000 & -.2928 & .2637 \\
\hline & & 3.00 & .00496 & .06292 & 1.000 & -.2054 & .2153 \\
\hline & & 4.00 & .00378 & .06067 & 1.000 & -.1990 & .2066 \\
\hline & & 5.00 & .08707 & .08324 & .954 & -.1912 & .3653 \\
\hline & & 6.00 & .09151 & .13162 & .993 & -.3485 & .5315 \\
\hline & 3.00 & 1.00 & -.01950 & .08616 & 1.000 & -.3075 & .2685 \\
\hline & & 2.00 & -.00496 & .06292 & 1.000 & -.2153 & .2054 \\
\hline & & 4.00 & -.00118 & .06462 & 1.000 & -.2172 & .2149 \\
\hline & & 5.00 & .08211 & .08616 & .969 & -.2059 & .3701 \\
\hline & & 6.00 & .08655 & .13348 & .995 & -.3597 & .5328 \\
\hline & 4.00 & 1.00 & -.01832 & .08453 & 1.000 & -.3009 & .2643 \\
\hline & & 2.00 & -.00378 & .06067 & 1.000 & -.2066 & .1990 \\
\hline & & 3.00 & .00118 & .06462 & 1.000 & -.2149 & .2172 \\
\hline & & 5.00 & .08329 & .08453 & .965 & -.1993 & .3659 \\
\hline & & 6.00 & .08773 & .13244 & .994 & -.3550 & .5305 \\
\hline & 5.00 & 1.00 & -.10160 & .10195 & .963 & -.4424 & .2392 \\
\hline & & 2.00 & -.08707 & .08324 & .954 & -.3653 & .1912 \\
\hline & & 3.00 & -.08211 & .08616 & .969 & -.3701 & .2059 \\
\hline & & 4.00 & -.08329 & .08453 & .965 & -.3659 & .1993 \\
\hline & & 6.00 & .00444 & .14418 & 1.000 & -.4775 & .4864 \\
\hline & 6.00 & 1.00 & -.10605 & .14418 & .990 & -.5880 & .3759 \\
\hline & & 2.00 & -.09151 & .13162 & .993 & -.5315 & .3485 \\
\hline & & 3.00 & -.08655 & .13348 & .995 & -.5328 & .3597 \\
\hline & & 4.00 & -.08773 & .13244 & .994 & -.5305 & .3550 \\
\hline & & 5.00 & -.00444 & .14418 & 1.000 & -.4864 & .4775 \\
\hline
\end{tabular}

Fuente: Resultados de la investigación 


\section{Cuadro 8}

\section{Estadísticos descriptivos del múltiplo pasivo en moneda extranjera del pasivo total}

\begin{tabular}{|c|c|c|c|c|c|c|c|c|}
\hline \multicolumn{9}{|c|}{ PMEaPT. Descriptivos } \\
\hline & \multirow[t]{2}{*}{$\mathbf{N}$} & \multirow[t]{2}{*}{ Media } & \multirow{2}{*}{$\begin{array}{l}\text { Desviación } \\
\text { típica }\end{array}$} & \multirow{2}{*}{$\begin{array}{l}\text { Error } \\
\text { típico }\end{array}$} & \multicolumn{2}{|c|}{$\begin{array}{c}\text { Intervalo de } \\
\text { confianza para la } \\
\text { media al } 95 \% \\
\end{array}$} & \multirow[t]{2}{*}{ Mínimo } & \multirow[t]{2}{*}{ Máximo } \\
\hline & & & & & $\begin{array}{l}\text { Limite } \\
\text { inferior }\end{array}$ & $\begin{array}{l}\text { Límite } \\
\text { superior }\end{array}$ & & \\
\hline $\begin{array}{l}\text { 1. Telecomunica- } \\
\text { ciones }\end{array}$ & 42 & .4722 & .14557 & .02246 & .4269 & .5176 & .22 & .71 \\
\hline $\begin{array}{l}\text { 2. Produc. } \\
\text { consumo frecuente }\end{array}$ & 126 & .3069 & .25795 & .02298 & .2614 & .3523 & 0.00 & .75 \\
\hline 3. Materiales & 98 & .5682 & .21473 & .02169 & .5252 & .6113 & 0.00 & .90 \\
\hline 4. Industrial & 112 & .2476 & .26067 & .02463 & .1988 & .2964 & 0.00 & .86 \\
\hline $\begin{array}{l}\text { 5. Serv. y bienes } \\
\text { cons. no frecuente }\end{array}$ & 42 & .1231 & .07441 & .01148 & .0999 & .1463 & .00 & .27 \\
\hline 6. Salud & 14 & .0840 & .01847 & .00494 & .0733 & .0946 & .05 & .11 \\
\hline Total & 434 & .3416 & .26868 & .01290 & .3163 & .3670 & 0.00 & .90 \\
\hline
\end{tabular}

Fuente: Resultados de la investigación.

Para el caso del nivel del pasivo en moneda extranjera PMEaPT el estadístico de Levene es menor a 0.05 , por lo cual se rechaza la hipótesis de igualdad de varianzas y el nivel de significancia del estadístico F, es menor a .05, también se rechaza la hipótesis de igualdad de medias.

En el cuadro 9 se presentan la prueba de las diferencias del valor medio del ratio que mide el uso de pasivos en moneda extranjera entre los diferentes sectores, en este caso en la columna de significación casi todas las combinaciones tienen valor menor a 0.05 , lo cual corrobora que existe diferencias estadísticamente significativas en el nivel de uso de pasivos en moneda extranjera entre los diferentes sectores de la Bolsa Mexicana de Valores, incluso en el cuadro 8 se muestra que las empresas del sector materiales y telecomunicaciones son los que tienen mayores niveles de deudas en moneda extranjera.

\section{Conclusiones}

El peso mexicano se ha depreciado frente al dólar más del 30\% durante el último lustro. Con la finalidad de evaluar el impacto de la depreciación de la moneda nacional en las 32 empresas no financieras del Índice de Precios y Cotizaciones de la Bolsa Mexicana de Valores durante 2012-2015, se calcula- 
ron los múltiplos financieros que miden las pérdidas cambiarias, las ganancias cambiarias y la cantidad de pasivos en moneda extranjera de cada una de las 32 empresas que integran el IPC.

Posteriormente se calculó un ANOva por cada uno de los múltiplos financieros, donde se compararon los valores medios de cada uno de los múltiplos financieros: (1) pérdidas cambiarias, (2) ganancias cambiarias y (3) deuda en moneda extranjera, para los seis sectores de la BMV, con la finalidad de evaluar el impacto de la depreciación del peso mexicano en cada uno de los sectores.

\section{Cuadro 9}

Comparaciones entre los sectores de empresas de la BMV pasivos en moneda extranjera

\begin{tabular}{|c|c|c|c|c|c|c|c|}
\hline \multicolumn{8}{|c|}{ Variable dependiente: PMEaPT. Comparaciones múltiples } \\
\hline & \multirow{2}{*}{\multicolumn{2}{|c|}{ (I) Sector }} & \multirow{2}{*}{$\begin{array}{l}\text { Diferen- } \\
\text { cia de } \\
\text { medias } \\
\text { (I-J) }\end{array}$} & \multirow{2}{*}{$\begin{array}{l}\text { Error } \\
\text { típico }\end{array}$} & \multirow{2}{*}{ Sig. } & \multicolumn{2}{|c|}{$\begin{array}{c}\text { Intervalo de confian- } \\
\text { za al } 95 \% \\
\end{array}$} \\
\hline & & & & & & $\begin{array}{l}\text { Límite } \\
\text { inferior }\end{array}$ & $\begin{array}{l}\text { Límite } \\
\text { superior }\end{array}$ \\
\hline \multirow[t]{24}{*}{ Scheffé } & 1.00 & 2.00 & $.16536^{\star}$ & .03987 & .005 & .0321 & .2986 \\
\hline & & 3.00 & -.09600 & .04127 & .369 & -.2340 & .0420 \\
\hline & & 4.00 & $.22461 \star$ & .04049 & .000 & .0893 & .3600 \\
\hline & & 5.00 & $.34912^{\star}$ & .04883 & .000 & .1859 & .5124 \\
\hline & & 6.00 & $.38827 \star$ & .06906 & .000 & .1574 & .6191 \\
\hline & 2.00 & 1.00 & $-.16536^{\star}$ & .03987 & .005 & -.2986 & -.0321 \\
\hline & & 3.00 & $-.26136^{\star}$ & .03014 & .000 & -.3621 & -.1606 \\
\hline & & 4.00 & .05925 & .02906 & .528 & -.0379 & .1564 \\
\hline & & 5.00 & $.18376^{\star}$ & .03987 & .001 & .0505 & .3170 \\
\hline & & 6.00 & $.22291 \star$ & .06304 & .030 & .0122 & .4337 \\
\hline & 3.00 & 1.00 & .09600 & .04127 & .369 & -.0420 & .2340 \\
\hline & & 2.00 & $.26136^{\star}$ & .03014 & .000 & .1606 & .3621 \\
\hline & & 4.00 & $.32061 \star$ & .03095 & .000 & .2171 & .4241 \\
\hline & & 5.00 & $.44512^{\star}$ & .04127 & .000 & .3072 & .5831 \\
\hline & & 6.00 & $.48427 \star$ & .06394 & .000 & .2705 & .6980 \\
\hline & 4.00 & 1.00 & $-.22461^{\star}$ & .04049 & .000 & -.3600 & -.0893 \\
\hline & & 2.00 & -.05925 & .02906 & .528 & -.1564 & .0379 \\
\hline & & 3.00 & $-.32061^{\star}$ & .03095 & .000 & -.4241 & -.2171 \\
\hline & & 5.00 & .12451 & .04049 & .095 & -.0108 & .2599 \\
\hline & & 6.00 & .16366 & .06343 & .250 & -.0484 & .3757 \\
\hline & 5.00 & 1.00 & $-.34912^{\star}$ & .04883 & .000 & -.5124 & -.1859 \\
\hline & & 2.00 & $-.18376^{\star}$ & .03987 & .001 & -.3170 & -.0505 \\
\hline & & 3.00 & $-.44512^{\star}$ & .04127 & .000 & -.5831 & -.3072 \\
\hline & & 4.00 & -.12451 & .04049 & .095 & -.2599 & .0108 \\
\hline
\end{tabular}




\begin{tabular}{|l|l|l|l|l|l|l|}
\hline & 6.00 & .03915 & .06906 & .997 & -.1917 & .2700 \\
\hline \multirow{2}{*}{6.00} & 1.00 & $-.38827 \star$ & .06906 & .000 & -.6191 & -.1574 \\
\hline & 2.00 & $-.22291 \star$ & .06304 & .030 & -.4337 & -.0122 \\
\hline & 3.00 & $-.48427 \star$ & .06394 & .000 & -.6980 & -.2705 \\
\hline & 4.00 & -.16366 & .06343 & .250 & -.3757 & .0484 \\
\hline & 5.00 & -.03915 & .06906 & .997 & -.2700 & .1917 \\
\hline
\end{tabular}

Fuente: Resultados de la investigación.

Todos los sectores tuvieron pérdidas cambiarias, la prueba ANOvA demostró que existen diferencias estadísticamente significativas entre los seis sectores en el valor promedio del múltiplo que mide las pérdidas cambiarias en relación a la utilidad neta, el sector de telecomunicaciones mantiene el nivel más alto con un valor promedio de $28 \%$ y el sector de servicios y bienes de consumo frecuente posee los niveles más bajos con un valor promedio de $1.46 \%$, el valor promedio de todas las empresas del IPC tuvieron 5.41\% pérdidas cambiarias del total de la utilidad neta (véase cuadro 2).

En lo que se refiere a las ganancias cambiarias casi todos los sectores tuvieron un valor promedio casi el 10\% del total de las utilidades, y se encontró que no existe diferencia estadística en los valores medios del ratio que miden las ganancias cambiarias entre los seis sectores de la Bolsa Mexicana de Valores, se puede decir que todas las empresas tuvieron el mismo nivel de múltiplo de ganancias cambiarias. Esto puede interpretarse que prácticamente son pocas las cuentas por cobrar en moneda extranjera que estas empresas tienen registradas en sus activos, lo que refleja su eficiencia en la recuperación de la cartera, pero a su vez no logran obtener beneficios de ganancias cambiarias en un entorno donde el peso mexicano se deprecia.

Finalmente en lo que se refiere al múltiplo que mide los pasivos en moneda extranjera, se observa que el sector de materiales es el que tiene los niveles más altos con un promedio de $56.82 \%$, del total de pasivo, el sector salud es el que mantienen el rango más bajo con el $8.40 \%$ del total de pasivos. Resulta interesante observar que pese a que todas las empresas mantienen pasivos en moneda extranjera, las pérdidas cambiarias apenas alcanzan los niveles de $5.41 \%$ del total de las utilidades netas (véase cuadro 2 y 8). Asimismo se encontraron que los resultados de la prueba ANOVA, señalan que existen diferencias estadísticamente significativas del nivel de deuda en moneda extranjera empleado por los sectores, sin embargo el sector telecomunicaciones y el de materiales son similares, asi como el de productos de consumo frecuente con el industrial. 
En esta investigación se evidencia que la depreciación del peso mexicano afecto en mayor medida a las empresas del sector telecomunicaciones, por las pérdidas cambiarias de $28.4 \%$ registradas en sus estados financieros, el sector materiales que tiene los niveles más altos de pasivos en moneda extranjera, alcanzando un nivel promedio 56\%, tan solo tuvieron pérdidas cambiarias del $8.5 \%$. Asimismo todas las empresas registraron ganancias cambiarias, las cuales son resultado de los ingresos en moneda extranjera por sus operaciones comerciales con el extranjero.

\section{Bibliografía}

Consejo Mexicano para la Investigación y Desarrollo de Normas de Información Financiera A.C., CINIF, (2015). NIF B-2, México, CINIF-IMCP.

Guzmán Ma. De la Paz, Leyva Soraya y Antonio Cárdenas. (2007). "La relación de causalidad entre el índice bursátil mexicano y el tipo de cambio spot”, Análisis Económico, Núm. 51, vol. XXII. Tercer cuatrimestre, pp. 81-105.

Guzmán María de la Paz y Padilla Ricardo. (2009). "El impacto de la política monetaria sobre la tasa de interés, el tipo de cambio y el índice bursátil”, Análisis Económico, Núm. 55, vol. XXIV. Primer cuatrimestre, pp. 47-76.

Haeussler Ernest, Paul Richard y Wood Richard, (2008), Matematicas para la administración y economía, México, Pearson Prentice Hall, pp. 799.

Hair Joseph, Anderson Rolph, Tatham Ronald y Black William, (1999), Análisis multivariante, España, Pearson Prentice Hall, pp. 799.

Kohler Heinz, (1998), Estadística para negocios y economía, México, CECSA, pp. 1053.

Krugman Paul y Wells Robin, (2013), Microeconomía, España, Reverté S A., pp. 595. López Francisco, Rodríguez Domingo y Ortiz Francisco (2011). "Volatilidad estocástica del tipo de cambio peso-dólar: el régimen flotante en México", Investigación económica, Núm. 276, vol. LXX, abril-junio, pp. 19-50.

Madura Jeff, (2015), Administración financiera internacional, México, CENGAGUE Learning., pp. 722.

Mankiw N. Gregory, (2015), Microeconomía. Versión para América Latina, México, CENGAGUE Learning., pp. 504.

Marín José y Rubio Gonzalo, (2001), Economía financiera, España, Antonio Bosch, pp. 984.

Nájera, María de Lourdes y Gutiérrez Raúl (2013). "Evolución del tipo de cambio peso mexicano/dólar estadounidense y el uso de derivados financieros”, Análisis Económico, Núm. 67, vol. XXVIII, primer cuatrimestre, pp. 153-170. 
- $\square=\square$ José Antonio Morales Castro, María Fernanda Velázquez Chávez y Cinthia Elvia García Rodríguez

Pérez López Cesar (2004), Técnicas de análisis multivariante de datos. Aplicaciones con SPSS, España, Pearson Prentice Hall, pp. 646.

Reyes Gerardo, Moslares Carlos y Sotomayor Jesús G. (2012). "Efectos de la volatilidad cambiaria en la Balanza Comercial: 2002-2010", Revista de la Facultad de Economía BUAP, Núm. 46, año. XVII, septiembre-diciembre, pp. 27-49.

Sosa Miriam y Cabello Alejandra, (2015). "Comportamiento bursátil en los G-9 emergentes (BRICs+4)”, Revista Problemas del Desarrollo, 181, 46, abril-junio, pp. 127-156.

\section{Fuentes especializadas y sitios web}

Economatica

Bolsa Mexicana de Valores: bolsamexicanadevalores.com.mx 